\title{
A NOTE ON THE THUE INEQUALITY
}

\author{
MICHAEL A. BEAN \\ (Communicated by William Adams)
}

\begin{abstract}
We show that for an arbitrary binary form $F(X, Y)$, there is no non-trivial lower bound for the area of the region $|F(x, y)| \leq 1$ which depends only on the discriminant of $F$.
\end{abstract}

\section{INTRODUCTION}

Let $F(X, Y)=a_{0} X^{n}+a_{1} X^{n-1} Y+\cdots+a_{n} Y^{n}$ be a binary form with complex coefficients and let $A_{F}$ denote the area of the region $|F(x, y)| \leq 1$ in the real affine plane. Let $D_{F}$ denote the discriminant of $F$. If $F$ has the factorization $\prod_{i=1}^{n}\left(\alpha_{i} X-\beta_{i} Y\right)$ with $\alpha_{i}, \beta_{i} \in \mathbb{C}$ (every binary form with complex coefficients has such a factorization), then $D_{F}=\prod_{i<j}\left(\alpha_{i} \beta_{j}-\alpha_{j} \beta_{i}\right)^{2}$. then

In [4], we showed that if $F$ has degree $n \geq 3$ and discriminant $D_{F} \neq 0$,

$$
\left|D_{F}\right|^{1 / n(n-1)} A_{F} \leq 3 B\left(\frac{1}{3}, \frac{1}{3}\right)
$$

where $B\left(\frac{1}{3}, \frac{1}{3}\right)$ denotes the Beta function with arguments of $1 / 3$. From this, we deduced that $A_{F} \leq 3 B\left(\frac{1}{3}, \frac{1}{3}\right)$ when $F$ is such a form with integer coefficients (since $D_{F} \in \mathbb{Z}$ in this case). The bound $3 B\left(\frac{1}{3}, \frac{1}{3}\right)$, which has approximate numerical value 15.8997 , is attained in both these inequalities when $F(X, Y)=X Y(X-Y)$.

As noted in [4], this result has significant implications in the enumeration of solutions of Thue inequalities. A Thue inequality is a Diophantine inequality of the type $|F(x, y)| \leq h$ where $F$ is a binary form with integer coefficients which is irreducible and has degree $n \geq 3$, and $h$ is a non-zero integer. In a seminal paper of 1909, Thue [11] showed that for such a form $F$, the equation $F(x, y)=h$ (hence the inequality $|F(x, y)| \leq h)$ has only a finite number of solutions in integers $x$ and $y$. In 1934, Mahler [7] showed that the number $N_{F}(h)$ of solutions of the Thue inequality $|F(x, y)| \leq h$ and the area $A_{F} h^{2 / n}$ of this region are related in the following way:

$$
\left|N_{F}(h)-A_{F} h^{2 / n}\right| \leq c h^{1 /(n-1)}
$$

Received by the editors October 26, 1993.

1991 Mathematics Subject Classification. Primary 11D75, 11J25.

Supported in part by a Postdoctoral Fellowship from the NSERC.

(c) 1995 American Mathematical Society 
where $c$ is a number (left unspecified by Mahler) which depends only on $F$. Estimates for $N_{F}(h)$ involving $A_{F} h^{2 / n}$ have also been given by Mueller and Schmidt [9]. Recently, Thunder [12] applied (1) to prove a special case of a conjecture of Schmidt.

In view of (1) and its application, Julia Mueller asked the author whether it is possible to obtain a positive absolute lower bound for $\left|D_{F}\right|^{1 / n(n-1)} A_{F}$. In this note, we will show that there is no such lower bound for $\left|D_{F}\right|^{1 / n(n-1)} A_{F}$ even over the class of forms with integer coefficients. In fact, we will show that in general, an upper bound on the size of either one of the quantities $A_{F}$ or $\left|D_{F}\right|$ does not yield a positive lower bound on the other.

\section{MAIN RESUltS}

If $F$ is a binary cubic form with real coefficients and non-zero discriminant, then $\left|D_{F}\right|^{1 / 6} A_{F} \geq \sqrt{3} B\left(\frac{1}{3}, \frac{1}{3}\right)$; in fact,

$$
\left|D_{F}\right|^{1 / 6} A_{F}= \begin{cases}3 B\left(\frac{1}{3}, \frac{1}{3}\right), & \text { if } D_{F}>0 \\ \sqrt{3} B\left(\frac{1}{3}, \frac{1}{3}\right), & \text { if } D_{F}<0\end{cases}
$$

(see [2]). However, for $n \geq 4$, there is no positive absolute lower bound for $\left|D_{F}\right|^{1 / n(n-1)} A_{F}$. Put $M_{n}=\max \left|D_{F}\right|^{1 / n(n-1)} A_{F}$ where the maximum is taken over all forms of degree $n$ with complex coefficients and $D_{F} \neq 0$.

Proposition. Fix $n \geq 4$. Over the class of forms of degree $n$ with real coefficients, the quantity $\left|D_{F}\right|^{1 / n(n-1)} A_{F}$ assumes all real values between 0 and $M_{n}$.

Proof. The quantity

$$
\left|D_{F}\right|^{1 / n(n-1)} A_{F}=\left|D_{F}\right|^{1 / n(n-1)} \int_{-\infty}^{\infty} \frac{d v}{|F(1, v)|^{2 / n}}
$$

is continuous in the roots of $F(1, y)$, and is maximized by a form with real coefficients for which $F(1, y)$ has $n$ distinct real roots (Theorem 3, [4]). Hence, we need only exhibit a sequence of forms $G_{N}$ such that $\left|D_{G_{N}}\right|^{1 / n(n-1)} A_{G_{N}} \rightarrow 0$.

In Theorem 3 of [3], we showed that if $F(X, Y)=\prod_{i=1}^{n}\left(\alpha_{i} X-\beta_{i} Y\right)$, where the $\alpha$ 's and $\beta$ 's are real numbers such that $\alpha_{1} / \beta_{1}<\cdots<\alpha_{n} / \beta_{n}$, then

$$
\left|D_{F}\right|^{1 / n(n-1)} A_{F}=\sum_{k=1}^{n} D_{F_{k}}^{1 / n(n-1)} \int_{0}^{1} \frac{d t}{F_{k}(1, t)^{2 / n}}
$$

where

$$
F_{k}(X, Y)=X Y(X-Y)\left(X-u_{1}^{(k)} Y\right) \cdots\left(X-u_{n-3}^{(k)} Y\right)
$$

and

$$
u_{j}^{(k)}=\frac{\left(\alpha_{k+1} \beta_{k}-\alpha_{k} \beta_{k+1}\right)\left(\alpha_{k-1} \beta_{k-1-j}-\alpha_{k-1-j} \beta_{k-1}\right)}{\left(\alpha_{k+1} \beta_{k-1}-\alpha_{k-1} \beta_{k+1}\right)\left(\alpha_{k} \beta_{k-1-j}-\alpha_{k-1-j} \beta_{k}\right)}
$$

for $1 \leq j \leq n-3,1 \leq k \leq n$, with the subscripts taken modulo $n$. In particular, $0<u_{1}^{(k)}<u_{2}^{(k)}<\cdots<u_{n-3}^{(k)}<1$, for each $k$.

Let $G_{N}$ be the form defined by putting $\alpha_{i}=N^{i}$ and $\beta_{i}=1(N>1)$. Then, with these values of $\alpha_{i}$ and $\beta_{i}$, we have $u_{j}^{(1)} \rightarrow 0, u_{j}^{(n)} \rightarrow 1$, and for $k \neq 1, n$

$$
u_{j}^{(k)} \rightarrow \begin{cases}0, & \text { if } k-j-1>0 \\ 1, & \text { if } k-j-1 \leq 0\end{cases}
$$


as $N \rightarrow \infty$; for example,

$$
u_{j}^{(n)}=\frac{\left(N-N^{n}\right)\left(N^{n-1}-N^{n-1-j}\right)}{\left(N-N^{n-1}\right)\left(N^{n}-N^{n-1-j}\right)}
$$

and the dominant term in both the numerator and denominator is $N^{2 n-1}$. We will show that, in general,

$$
\begin{aligned}
& D_{F_{k}}^{1 / n(n-1)} \int_{0}^{1} \frac{d t}{F_{k}(1, t)^{2 / n}} \\
& \quad<B\left(1-\frac{2}{n}, \frac{1}{n(n-1)}\right) \prod_{j=1}^{n-3}\left(u_{j}^{(k)}\left(1-u_{j}^{(k)}\right)\right)^{1 / n(n-1)(n-3)} .
\end{aligned}
$$

It will then follow that $\left|D_{G_{N}}\right|^{1 / n(n-1)} A_{G_{N}} \rightarrow 0$ as $N \rightarrow \infty$.

To simplify notation, let us suppose that $k=1$ and write $u_{j}=u_{j}^{(1)}$. Using the estimates, $u_{j}<1$, and $\left|u_{l}-u_{j}\right|<1-u_{j}$ for $j<l$, we have

$$
D_{F_{1}}^{1 / n(n-1)}<\prod_{j=1}^{n-3}\left(u_{j}^{1 /(n-3)}\left(1-u_{j}\right)^{2(n-j-2)}\right)^{1 / n(n-1)} .
$$

Put $\varepsilon=1 / n(n-1)(n-3)$ and $q=\prod_{j=1}^{n-3} u_{j}^{\varepsilon}\left(1-u_{j}\right)^{\varepsilon}$. Then, using the estimates $1-u_{j}<1-u_{j} t$ and $\left(1-u_{j} t\right)^{-1}<(1-t)^{-1}$ we have

$$
\begin{aligned}
D_{F_{1}}^{1 / n(n-1)} \int_{0}^{1} \frac{d t}{F_{1}(1, t)^{2 / n}} & <q \int_{0}^{1} \frac{\prod_{j=1}^{n-3}\left(1-u_{j} t\right)^{2((n-j-2) / n(n-1))-\varepsilon} d t}{t^{2 / n}(1-t)^{2 / n} \prod_{j=1}^{n-3}\left(1-u_{j} t\right)^{2 / n}} \\
& =q \int_{0}^{1} \frac{\prod_{j=1}^{n-3}\left(1-u_{j} t\right)^{-2((j+1) / n(n-1))-\varepsilon} d t}{t^{2 / n}(1-t)^{2 / n}} \\
& \leq q \int_{0}^{1} \frac{d t}{t^{2 / n}(1-t)^{1-1 / n(n-1)}} \\
& =q B\left(1-\frac{2}{n}, \frac{1}{n(n-1)}\right) .
\end{aligned}
$$

This establishes (2).

Now from (2), it is clear that $D_{F_{k}}^{1 / n(n-1)} \int_{0}^{1} F_{k}(1, t)^{-2 / n} d t \rightarrow 0$ if either $u_{1}^{(k)} \rightarrow$ 0 or $u_{n-3}^{(k)} \rightarrow 1$. Consequently, $\left|D_{G_{N}}\right|^{1 / n(n-1)} A_{G_{N}} \rightarrow 0$ as $N \rightarrow \infty$.

Corollary. Fix $n \geq 4$. Over the class of forms of degree $n$ with integer coefficients, the quantity $\left|D_{F}\right|^{1 / n(n-1)} A_{F}$ assumes arbitrarily small positive values.

Proof. Since the forms $G_{N}$ of the preceding proof have integer coefficients when $N$ is a positive integer, it is clear that there is no positive absolute lower bound for $\left|D_{F}\right|^{1 / n(n-1)} A_{F}$ over the class of forms with integer coefficients even though the set of values $\left\{\left|D_{F}\right|^{1 / n(n-1)} A_{F}\right\}$ is countable in this case.

Remark. For the cubic forms $F^{(t)}(X, Y)=\left(X^{2}+Y^{2}\right)(Y-i t X), t \in(0,1)$, we have $A_{F^{(t)}}=\int_{0}^{1} z^{-1 / 2}(1-z)^{-1 / 2}\left(1-\left(1-t^{2}\right) z\right)^{-1 / 3} d z \leq B\left(\frac{1}{2}, \frac{1}{6}\right)$ and $\left|D_{F^{(t)}}\right|^{1 / 6}=$ 
$2^{1 / 3}\left(1-t^{2}\right)^{1 / 3}$; so $\left|D_{F^{(t)}}\right|^{1 / 6} A_{F^{(t)}} \rightarrow 0$ as $t \rightarrow 1$. Hence $\left|D_{F}\right|^{1 / 6} A_{F}$ assumes all values between 0 and $3 B\left(\frac{1}{3}, \frac{1}{3}\right)$ over the cubic forms with complex coefficients.

\section{Related EXAMPLES}

It is clear from (1) that if either of the quantities $\left|D_{F}\right|^{1 / n(n-1)}$ or $A_{F}$ is large, then the other must be small since a lower bound for one implies an upper bound for the other. It is natural to ask whether the converse situation holds; that is, whether an upper bound for one implies a lower bound for the other. In this section, we will give examples to show that this is not the case. We will also show that there are forms $F$ with integer coefficients and $D_{F}=0$ for which $A_{F}$ is arbitrarily large or arbitrarily small.

For any complex number $k, A_{k F}=|k|^{-2 / n} A_{F}$ and $\left|D_{k F}\right|=|k|^{2(n-1)}\left|D_{F}\right|$. Hence, by appropriately choosing $k$, we can ensure that either $A_{F}<1$ or $\left|D_{F}\right|<1$ as desired.

Example 3.1. Consider the forms

$$
F^{(t)}(X, Y)=k(X+i t Y)(X+i(t+1) Y) \cdots(X+i(t+n-1) Y), \quad t \in \mathbb{R} .
$$

Notice that $\left|D_{F^{(n)}}\right|^{1 / n(n-1)}=|k|^{2 / n}((n-1) !(n-2) ! \cdots 2 ! 1 !)^{2 / n(n-1)}$ which is independent of $t$, and that $A_{F^{(t)}}<4|k|^{-2 / n} t^{-1}$ since the region $\left|F^{(t)}(x, y)\right| \leq 1$ is contained in the region $|x| \leq|k|^{-1 / n},|y| \leq|k|^{-1 / n} t^{-1}$. Given $\varepsilon>0$, we can ensure that $\left|D_{F^{(t)}}\right|^{1 / n(n-1)}<\varepsilon$ for all $t$ by appropriately choosing $k$; then, letting $t \rightarrow \infty$, we have $A_{F^{(t)}} \rightarrow 0$ while $\left|D_{F^{(t)}}\right|^{1 / n(n-1)}<\varepsilon$. Hence, an upper bound for $\left|D_{F}\right|$ does not imply a lower bound for $A_{F}$.

Example 3.2. Consider the forms

$$
F^{(t)}(X, Y)=k(X+i t Y)(X+i Y)(X+2 i Y) \cdots(X+(n-1) i Y), \quad t \in(0,1) .
$$

In this case,

$$
\begin{aligned}
A_{F^{(t)}} & =|k|^{-2 / n} \int_{-\infty}^{\infty} \frac{d u}{\left|\left(u^{2}+t^{2}\right)\left(u^{2}+1\right)\left(u^{2}+2^{2}\right) \cdots\left(u^{2}+(n-1)^{2}\right)\right|^{1 / n}} \\
& \leq|k|^{-2 / n} \int_{-\infty}^{\infty} \frac{d u}{\left|u^{2}\left(u^{2}+1\right)\left(u^{2}+2^{2}\right) \cdots\left(u^{2}+(n-1)^{2}\right)\right|^{1 / n}} \\
& \leq|k|^{-2 / n} \frac{3 B\left(\frac{1}{3}, \frac{1}{3}\right)}{((n-1) !(n-2) ! \cdots 1 !)^{2 / n(n-1)}}
\end{aligned}
$$

which is independent of $t$; however, $\left|D_{F^{(t)}}\right| \rightarrow 0$ as $t \rightarrow 1$. Hence, an upper bound for $A_{F}$ does not imply a lower bound for $\left|D_{F}\right|$.

Example 3.3. Consider the binomial forms

$$
F(X, Y)=k X^{(n-1) / 2} Y^{(n-1) / 2}(X-Y)
$$

where $n$ is an odd integer greater than three and $k$ is a positive integer. In this case, $D_{F}=0, A_{F}<\infty$, and

$$
\begin{aligned}
A_{F} & =k^{-2 / n} \int_{-\infty}^{\infty} \frac{d v}{\left|v^{(n-1) / 2}(1-v)\right|^{2 / n}} \\
& >k^{-2 / n} \int_{0}^{1} \frac{d v}{v^{(n-1) / n}(1-v)^{2 / n}} \\
& =k^{-2 / n} B\left(\frac{1}{n}, 1-\frac{2}{n}\right) .
\end{aligned}
$$


Notice that $A_{F}$ can be made large by fixing $k$ and choosing $n$ sufficiently large; on the other hand, $A_{F}$ can be made small by fixing $n$ and choosing $k$ sufficiently large. Hence, when $D_{F}$ is zero, $A_{F}$ can be arbitrarily large or arbitrarily small even if we assume that $F(X, Y) \in \mathbb{Z}[X, Y]$.

Hence we have proved the following result.

Proposition. There do not exist positive numbers $C, \alpha$ for which

$$
A_{F} \geq \frac{C}{\left|D_{F}\right|^{\alpha}}
$$

for all forms $F$ of degree $n$ with $D_{F} \neq 0$. In particular, an upper bound for one of the quantities $A_{F},\left|D_{F}\right|$ does not imply a lower bound for the other. Moreover, when $D_{F}=0, A_{F}$ can be arbitrarily large or arbitrarily small even if we assume that $F(X, Y) \in \mathbb{Z}[X, Y]$.

\section{REFERENCES}

1. M. Abramowitz and I. Stegun, Handbook of mathematical functions, Dover, New York, 1965.

2. M. A. Bean, Areas of plane regions defined by binary forms, Ph.D. thesis, Univ. of Waterloo, 1992.

3. __ Binary forms, hypergeometric functions, and the Schwarz-Christoffel mapping formula, Trans. Amer. Math. Soc. (to appear).

4. __ An isoperimetric inequality for the area of plane regions defined by binary forms, Compositio Math. 92 (1994), 115-131.

5. M. A. Bean and J. L. Thunder, Isoperimetric inequalities for volumes associated with decomposable forms, J. London Math. Soc. (to appear).

6. G. Hardy, J. E. Littlewood, and G. Polya, Inequalities, Cambridge Univ. Press, London and New York, 1952.

7. K. Mahler, Zur Approximation algebraischer Zahlen III, Acta Math. 62 (1934), 91-166.

8. J. Mueller and W. M. Schmidt, On the Newton polygon, Monatsh. Math. 113 (1992), 33-50.

9. Thue's equation and a conjecture of Siegel, Acta Math. 160 (1988), 207-247.

10. W. M. Schmidt, Diophantine approximations and Diophantine equations, Lecture Notes in Math., vol. 1467, Springer-Verlag, New York, 1991.

11. A. Thue, Über Annäherungswerte algebraischer Zahlen, J. Reine Angew. Math. 135 (1909), 284-305.

12. J. L. Thunder, The number of solutions to cubic Thue inequalities, Acta. Arith. LXVI (1994), 237-243.

Department of Mathematics, University of Toronto, Toronto, Ontario, Canada M5S 1A1 48109

Current address: Department of Mathematics, University of Michigan, Ann Arbor, Michigan

E-mail address: mbean@math.1sa.umich.edu

E-mail address: bean@math.toronto.edu 